\title{
03 BRIDGING THE GAP BETWEEN RESEARCH EVIDENCE AND REAL-WORLD IMPLEMENTATION: THE NOGAPS PROJECT
}

doi:10.1136/injuryprev-2012-040590g.3

${ }^{1} \mathrm{~B}$ Gabbe, ${ }^{2} \mathrm{~J}$ Cook, ${ }^{3} \mathrm{D}$ Lloyd, ${ }^{4} \mathrm{~A}$ Donaldson, ${ }^{5} \mathrm{~W}$ Young, ${ }^{4} \mathrm{C}$ Finch. ${ }^{1}$ Department of Epidemiology and Preventive Medicine, Monash University, Australia; ${ }^{2}$ Department of Physiotherapy, Monash University, Australia; ${ }^{3}$ School of Rehabilitation Sciences, Griffith University, Australia; ${ }^{4}$ Australian Centre for Research into Injury in Sport and its Prevention, Monash University, Australia; ${ }^{5}$ School of Human Movement and Sport Sciences, University of Ballarat, Australia

Background There is an increasing momentum to develop, widely implement and evaluate evidence-based injury prevention programmes in sport. However, navigating the literature to evaluate the evidence, and bridging the gap between the research environment and the real-world implementation context, is challenging.

Aims/Objectives/Purpose This study aimed to develop an evidence-based lower limb injury prevention exercise programme for implementation and evaluation in community Australian football.

Methods Development of the programme involved a systematic review of the literature including consideration of compliance, cost, adverse effects, uptake and feasibility. The review was used to develop a draft exercise-based programme (FootyFirst) to prevent hamstring, hip/groin, knee and ankle injuries. The programme was presented to stakeholders in Australian football for feedback through a Delphi process and then field tested for feasibility. The final programme was then implemented in two football leagues under two conditions (fully supported and programme only), with a third league acting as a control.

Results/Outcome The evidence-base for lower limb injury prevention strategies was limited, making direct extrapolation of research findings to programme content challenging. Three rounds of the Delphi process were required before consensus of the programme content was achieved. Further modifications were made after field testing. The programme was implemented in time for the 2012 season.

Significance/Contribution to the Field Developing a comprehensive programme with a strong foundation in the published research evidence was challenging, requiring many modifications and extensive consultation to develop a programme with face validity and a strong probability of uptake. The effectiveness FootyFirst is currently being evaluated. 